\title{
Estudio de caso a usuario con diagnostico: síndrome de vena cava superior por neoplasia en mediastino
}

\author{
Velázquez-Ahumada, Ricardo*; Escalante-Verdugo, Manuela de Jesús²
}

\begin{abstract}
RESUMEN
Introducción: El síndrome de la vena cava superior es una entidad grave generada por la obstrucción del flujo sanguíneo, puede generarse por presión extrínseca o por invasión de la misma, el cuidado de enfermería a estos usuarios se centra en actuar sobre la respuesta clínica que manifiesta el compromiso del estado de salud del usuario. Objetivo: Presentar una propuesta de cuidado centrada en el estado de salud del usuario. Metodología: Se realizó un estudio de caso bajo la metodología del proceso de enfermería de acuerdo con la tipología de patrones funcionales de salud de Marjory Gordon, con el fin de detectar las principales respuestas humanas del usuario. Se priorizaron los diagnósticos enfermeros de acuerdo con la taxonomía II de la NANDA-I y en base a juicios clínicos se elaboraron planes de atención de enfermería estableciendo resultados e intervenciones de acuerdo con las taxonomías NOC-NIC Presentación del caso: Usuario de 62 años ingresa a urgencias y se hospitaliza por presentar probable cuadro de neumonía. Posterior a estudios diagnósticos se traslada a al servicio de medicina interna con diagnóstico de Síndrome de vena cava superior por neoplasia de mediastino Conclusiones: Se aplicarion las cinco etapas del proceso de enfermería, lo que se reflejó en la evolucion postivia de la persona. El cuidado del paciente crítico se afecta por la falta de identificación temprana de alteraciones en el estado de salud lo cual impacta negativamente en el proceso salud-enfermedad.
\end{abstract}

Palabras claves: Proceso de enfermería; síndrome de la vena cava superior; neoplasias del mediastino; gastrostomía; estudio de caso (DeCS; BIREME).

\footnotetext{
${ }_{1}^{1}$ Pasante de Licenciatura en Enfermería. Departamento de Enfermería. Universodad de Sonora. E-mail: ricardo_27velazquez@hotmail.com

${ }^{2}$ Maestra en Educación Basada en Competencias. Docente de Asignatura. Departamento de Enfermería. Universidad de Sonora. Área de Enseñanza e Investigación de Enfermería. Hospital General del Estado de Sonora "Dr. Ernesto Ramos Bours". E-mail: mescalanteverdugo@gmail.com
}

*Autor para correspondencia

Cómo citar este artículo

Velázquez-Ahumada R, Escalante-Verdugo MJ. Estudio de caso a usuario con diagnostico: síndrome de vena cava superior por neoplasia en mediastino. Sanus. 2018;3(7): 78-95. [Acceso ]; Disponible en: mes día año 


\section{INTRODUCCIÓN}

El proceso de enfermería (PE) se define como un método sistemático de solución de problemas para brindar cuidados de enfermería al individuo, familia o comunidad. Constituye la base de las acciones de enfermería y puede aplicarse en cualquier ámbito de atención de salud o de tipo educativo, en cualquier marco de referencia teórico o conceptual, y en el contexto de cualquier filosofía de la enfermería ${ }^{(1)}$. El objetivo del PE es identificar el estado de salud del usuario y/o comunidad, así como los problemas reales y potenciales, de esta manera establecer planes de atención en torno a las necesidades identificadas, realizando intervenciones de enfermería para resolverlas, lo que requiere de pensamiento crítico para su correcta realización ${ }^{(2)}$.

El estudio de caso se basa en describir una circunstancia del entorno tomada en su contexto y en estudiarla con el fin de analizar cómo se manifiestan y evolucionan los fenómenos de interés. Se describe el caso como relevante de acuerdo a la viabilidad para explicar un fenómeno, donde el contexto es determinante. Es relevante para enfermería puesto que uno de los componentes involucrados en el metaparadigma de enfermería es el entorno y las afecciones que tienen sobre el usuario en el proceso salud-enfermedad.

El estudio de caso es de interés para la disciplina de enfermería ya que permite la reflexión y el análisis sobre situaciones clínicas que podemos utilizar como fuentes de aprendizaje, así mismo como propuestas de mejora, impactando de manera positiva en la atención otorgada al usuario ${ }^{(3)}$.

El síndrome de la vena cava superior (SVCS) es un cuadro clínico grave por la obstrucción del flujo sanguíneo de la vena cava superior (VCS) dirigido a la aurícula derecha, el cual puede generarse por presión extrínseca o por invasión de la misma. Su diagnóstico prácticamente es clínico, caracterizado por síntomas específicos como la disnea, igual de importantes los hallazgos relacionados a la exploración física; como principales están el edema facial y la distensión venosa del cuello junto con la pared torácica. El desarrollo normalmente es de causa maligna en el $90 \%$ de los $\operatorname{casos}^{(4)}$.

El diagnóstico de neoplasia en mediastino se realiza en base a la clínica presentada.

1. El $60 \%$ de los enfermos no presentan síntomas y recurren a la atención médica por el descubrimiento de una anormalidad radiográfica.

2. Existen síntomas respiratorios como tos seca irritativa o con expectoración mucosa, disnea, dolor torácico, estridor, fragmentos de calcio, sangre por erosión del tumor o la masa en vías aéreas.

3. Síndrome de vena cava superior, por deterioro en el vaciado de las venas cefálicas, cuello y extremidades superiores. Si la obstrucción es rápida, predispone a la aparición de ingurgitación venosa, edema y cianosis en cabeza, cuello, tronco y miembros superiores

4. Síndrome de Horner: Por participación de las ramas 6 ta cervical y 1 ra torácica, donde se puede observar pupilas mióticas y depreciación de la curvatura palpebral por presencia de ptosis, a veces acompañada de enoftalmos y trastornos de la hidratación o temperatura de la hemicara afectada.

5. Síndrome febril. Puede desarrollarse hasta sepsis, como en patologías relacionadas a linfoma, masa infecciosa por mycobacterias, hongos o bacterias, necrosis o infección de la masa o infección en región alejada a la compresión, lo que termina en el desarrollo de neumonía, absceso y síndrome de supuración pulmonar, con secreciones infecciosas y olor desagradable.

6. Otros síntomas y signos; Estridor traqueal, arritmias cardíacas, quilotórax, disfonía, disfagia, odinofagia, compresión medular de raíces, arterias y otras venas independientes a la cava superior, derrame pericárdico, insuficiencia cardiaca, así como síndrome de pancoast.

7. Identificación por marcadores tumorales: elevación de anticuerpos antirreceptor de acetilcolina, Alfafetoproteina, Fracción beta de gonadotropina coriónica ${ }^{(5)}$.

Siendo causas principales de neoplasia mediastinal: cáncer de pulmón no microcítico (CPNM) en la mayoría de los casos, en segundo lugar el cáncer de pulmón de células pequeñas (CPCP) y linfoma no Hodgkin $(\mathrm{LNH})$. Otros tumores malignos de mínima incidencia que se pueden presentar son el timoma, la neoplasia mediastínica de células germinales, el mesotelioma y las metástasis (4).

El presente estudio de caso tiene como propósito presentar una propuesta de cuidado a un usuario con diagnóstico médico de síndrome de vena cava superior por neoplasia en mediastino, post-operado de gastrostomía, al que se le realizó el PE de acuerdo a la tipología para valoración de patrones funcionales de salud de Marjory Gordon.

\section{METODOLOGÍA}

El estudio de caso se realizó en el área de Medicina Interna de una institución de seguridad pública de segundo nivel. Se utilizó la metodología del proceso de enfermería según tipología de patrones funcionales de la salud de Marjory Gordon. Para la recolección de datos, se interrogó al familiar ya que las condiciones del usuario no permitían interrogarlo de forma directa, además se comparó la información obtenida con la recopilada en expediente clínico.

El abordaje se dio a través del proceso de enfermería, que está constituido por 5 etapas: valoración, diagnóstico, planeación, ejecución y evaluación. Se identificaron diagnósticos enfermeros de acuerdo a taxonomía II de la NANDA-I. Posterior a ello se planearon 
los cuidados de enfermería de acuerdo a taxonomía NOC-NIC, estableciendo resultados esperados de acuerdo a las intervenciones establecidas. Asimismo se realizó revisión bibliográfica y de artículos científicos para analizar la información obtenida del usuario y de esta forma tener un panorama más acorde a su estado de salud.

\section{PRESENTACIÓN DEL CASO}

\section{Antecedentes de ingreso}

Hombre de 62 años, reside en Hermosillo, Sonora. Ingresa al servicio de urgencias por su propio pie, refiriendo disfagia y disfonías así como picos febriles, por lo cual se decide internar por posible neumonía. Previo a su hospitalización el usuario acudió a consulta médica por referir acidez estomacal y ligera opresión en el pecho, con tratamiento establecido sin presentar mejoría.

Como método diagnóstico se realiza una tomografía computarizada (TC) y estudios sanguíneos, posterior es trasladado al servicio de medicina interna con diagnóstico de síndrome de vena cava superior por neoplasia en mediastino.

Durante la valoración de enfermería, la entrevista fue realizada a los familiares del usuario debido al estado de criticidad y estado de conciencia.

\section{Antecedentes personales}

Patológicos y tratamientos recibidos: Familiar niega enfermedades crónicas.

No patológicos: Esquema de vacunación incompleto, alergias negadas.

Heredo-Familiares: Madre fallece por peritonitis a los 75 años. Padre fallece por sangrado de tubo digestivo bajo a los 65 años, hermano finado a los 55 años por CA de estómago.

\section{Indicaciones Médicas}

Soluciones parenterales:

-Nutrición parenteral $\mathrm{n} 7 \mathrm{de} 2000 \mathrm{ml}$ a $41.6 \mathrm{ml} / \mathrm{h}$

- Solución $\mathrm{NaCl}$ al 0.9\% $200 \mathrm{ml}+200 \mathrm{mg}$ de Midazolam

a $25.0 \mathrm{ml} / \mathrm{h}$

- Solución $\mathrm{NaCl}$ al 0.9\% $60 \mathrm{ml}+2$ mg de Fentanilo a 12 $\mathrm{ml} / \mathrm{hrs}$.

- PFC $200 \mathrm{ml} \mathrm{c} / 6$ hrs (10 am, 4pm, 10pm, 4 am)

\section{Medicamentos:}

- Furosemide 20mg (2ml) I.V c/8 hrs (10am, 6pm, 2 am)

- Omeprazol 40 mg (diluido en 10 ml S.F) I.V c/24 (10 am)

- Dexametasona 4 mg (1ml) I.V c/24 h (12 pm)

- Ertapenem 1 gr (Diluido en 100 de SF) I.V. c/24h (12 pm)

- Ácido Fólico 1mg (10ml) S.G. c/24 (10 am)

- Sucralfato $1 \mathrm{gr}$ (10ml) S.G. c/6 h (6am, 12pm, 6pm, 12am.)

\section{Valoración y documentación por patrones funcionales de Margory Gordon}

\section{Patrón mantenimiento-percepción de la salud}

Anterior a su hospitalización presentaba buen estado de salud, sin embargo según refiere familiar en el último mes se ha deteriorado considerablemente por notar pérdida de peso, cansancio generalizado, dificultad respiratoria. Tabaquismo positivo con un consumo de seis cigarros diarios desde los 18 años + consumo de alcohol ocasional a razón de 2 litros. Sin presencia de enfermedades crónico degenerativas. Acude a consulta médica cada seis meses. Vivienda con equipo y mobiliario acorde a las necesidades básicas según lo expresado por el familiar. Se consideró este patrón como disfuncional.

\section{Patrón Nutricional-Metabólico}

Usuario de complexión robusta, tez morena, presencia de edema con Godette +++ en extremidades superiores. Caries en 20 de 28 piezas dentarias, halitosis, lesiones con sangrado en cavidad oral y edema labial debido a tubo orotraqueal. Presenta un IMC de $29.04 \mathrm{~kg} / \mathrm{m}^{2}$ (sobrepeso), sin embargo, existe pérdida de peso de $14 \mathrm{~kg}$ aproximadamente en el último mes. Peristaltismo aumentado de $25 x^{\prime}$, ruidos hipersonoros, perímetro abdominal prepandial de $118 \mathrm{~cm}$ y postprandial de $122 \mathrm{~cm}$. Gastrostomía funcional sin datos de infección en zona de inserción, Cuenta con nutrición enteral de tres veces al día de $220 \mathrm{ml}$ siendo esta dieta licuada, además nutrición parenteral $\mathrm{N} 7$ de $2000 \mathrm{~mL}$ p/48 hrs. Normotérmico, . Ingreso de $946 \mathrm{ml}$ durante el turno de 5 hrs (Vía enteral: $220 \mathrm{ml}$, parenteral: $726 \mathrm{ml}$ ), con tendencia a la hiperglicemia.

Tegumentos sub hidratados, presenta úlcera por presión en región sacro-coccígea clasificada en estadio 2, de aproximadamente $4 \mathrm{~cm}$ de longitud, en fase proliferativa, la cual se encuentra cubierta con gasa, sin datos de infección.

Los resultados de laboratorio muestran leucocitosis y neutrofilia y anemia (Tabla 1), relacionado a la enfermedad de base de neoplasia de mediastino según lo establecido por la bibliografía ${ }^{(4)}$. Por lo mencionado, este patrón se encuentra disfuncional.

\section{Patrón de eliminación}

Cuenta con sonda vesical funcional, con uresis de 58.4 $\mathrm{ml} \mathrm{x} \mathrm{hr}$, con diuresis horaria de $0.64 \mathrm{~mL} / \mathrm{kg} / \mathrm{h}$ (oliguria), y un total de $292 \mathrm{ml}$ en el turno, apariencia amarilla clara. Meato urinario íntegro. Presencia de estreñimiento (10 días sin evacuación intestinal). Pérdidas insensibles de 225 por turno (90 kg x $0.5 \times 5$ horas), balance hídrico positivo de $+429 \mathrm{ml}$. 
Tabla 1. Resultados de laboratorio del patrón nutricional-metabólico

\begin{tabular}{|l|l|l|}
\hline EXAMEN & VALOR DE REFERENCIA & CIFRAS DEL USUARIO \\
\hline \multicolumn{2}{|l|}{ Biometría Hemática (10/08/16) } & $\mathrm{O}+$ \\
\hline Tipo y Rh & & $12.910^{\wedge} / \mathrm{uL}$ \\
\hline Leucocitos & $4.6-10.2$ & $2.9910^{\wedge} 6 / \mathrm{uL}$ \\
\hline Eritrocitos & $4.20-6.40$ & $8.3 \mathrm{~g} / \mathrm{dL}$ \\
\hline Hemoglobina & $12.2-18.1$ & 26.7 \\
\hline Hematocrito & $36-52$ & $13610^{\wedge 3} / \mathrm{uL}$ \\
\hline Plaquetas & $150-500$ & $85.6 \%$ \\
\hline Neutrófilos & $45.0-70.0 \%$ & $5 \%$ \\
\hline Linfocitos & $15.0-45.0 \%$ & \\
\hline Química Sanguínea & $75-110 \mathrm{mg} / \mathrm{dL}$ & 96 \\
\hline Glucosa & & \\
\hline
\end{tabular}

Fuente: Expediente clínico

Tabla 2. Resultados de laboratorio del patrón de eliminación

\begin{tabular}{|c|c|c|}
\hline EXAMEN & VALOR DE REFERENCIA & CIFRAS DEL USUARIO \\
\hline \multicolumn{3}{|l|}{ EGO (10/08/16) } \\
\hline Color & & Amarillo-aspecto claro \\
\hline Densidad Específica & $1010-1.030 \mathrm{~g} / \mathrm{L}$ & $1.005 \mathrm{~g} / \mathrm{L}$ \\
\hline $\mathrm{PH}$ & 5-6.5 & 5 \\
\hline Leucocitos & & Negativos \\
\hline Nitritos & & Negativos \\
\hline Proteínas & Negativo & Negativo \\
\hline Glucosa & Negativo & negativo \\
\hline Cetonas & Negativo & negativo \\
\hline Urobilinogeno & & Normal \\
\hline Bilirrubinas & & Negativo \\
\hline Sangre & & Negativo \\
\hline Células epiteliales & & Escaso \\
\hline Bacterias & & Escaso \\
\hline Hematíes & & 0.1 \\
\hline Filamentos mucosos & & Escasos \\
\hline Uratos & & Escasos \\
\hline \multicolumn{3}{|c|}{ Bioquímica $(10 / 8 / 16)$} \\
\hline Urea & 16.6-48.5 & 29 \\
\hline Creatinina & $0.7-1.2 \mathrm{mg} / \mathrm{dL}$ & 0.61 \\
\hline
\end{tabular}

Fuente: Expediente clínico 
De acuerdo a laboratorios, presenta hipostenuria (Tabla 2) relacionado con la retención de líquido. Urea 29 mg/dL Creatinina 0.61 mg/dL) (Tabla 2) lo cual indica un aumento a la tasa filtración glomerular de $153 \mathrm{ml} / \mathrm{min}$ (140-62 x 90/ 75 x 0.61). Por lo anterior, se consideró este patrón como disfuncional.

\section{Patrón actividad y ejercicio}

Usuario con ventilación mecánica asistida-controlada con los siguientes parámetros: frecuencia respiratoria de 12 $x^{\prime}$ con ventilaciones espontaneas (1-2x'), PEEP $7 \mathrm{cmH} 2 \mathrm{O}$, relación I:E de 1:2.5, VTE 580 y FiO2 al 50\%. Campos pulmonares con presencia de estertores bilaterales en ápices pulmonares, con adecuada expansión torácica, $\mathrm{SaO} 2$ de 95\%. Presenta ingurgitación yugular bilateral. Tensión arterial de 117/80 mmHg (PAM: 92), frecuencia cardíaca de 82 x', rítmica, llenado capilar de 3s en miembros periféricos. Cuenta con acceso venoso central en vena femoral derecha de tres lúmenes, permeable, sin datos de infección, con 16 días de instalado.

Se observa alteración de la gasometría arterial lo que indica la presencia de acidosis respiratoria (Tabla 3), además una alteración en la ventilación/perfusión en el aporte de O2, Índice de kirby 169 (PaO2=76 /FiO2 45\%* 100).

EKG muestra FC de 84x', rítmico, con $\mathrm{P}$ pulmonale, de V1 a V3 muestra QRS con R prima por bloqueo de rama derecha, relacionado con afección pulmonar.

Tiempos de coagulación alargados (T.P. 18 s TPP. 45.8s). plaquetopenia (136 000 plaquetas) (Tabla 3). Debido a esto se considera como un patron disfuncional.

Patrón sueño - descanso

Patrón no valorado debido al estado de salud.

\section{Patrón cognitivo - perceptivo}

Usuario bajo sedación profunda con puntaje de -4 según escala de RASS.

Diámetro pupilar de $2 \mathrm{~mm}$ (miosis) con mínima reacción a la luz debido a la infusión con opioides.

Por lo anterior, se considera el patrón como disfuncional.

Patrón autopercepción y concepto de sí mismo

Patrón no valorado debido al estado de conciencia del usuario

\section{Patrón Rol-Relaciones}

Usuario vive con su hijastro, viudo, buena relación con sus hijastros.

\section{Patrón de Sexualidad - Reproducción}

No tiene hijos, se desconoce vida sexual.

Mamas integras tipo I. Presenta genitales íntegros sin presencia de infección, reflejo cremasteriano ausente, región perianal integra. Por lo que se considera el patrón como funcional.

\section{Patrón de afrontamiento-tolerancia al estrés}

Patrón no valorado debido al estado de conciencia del usuario.

\section{Patrón valores y creencias}

Patrón no valorado debido al estado de conciencia del usuario.

Tabla 3. Resultados de laboratorio del patrón actividad-ejercicio.

\begin{tabular}{|l|l|l|}
\hline EXAMEN & VALOR DE REFERENCIA & CIFRAS DEL USUARIO \\
\hline \multicolumn{2}{|l|}{ Gasometría Arterial (10/8/16 11:27 hrs) } & $76 \mathrm{mmHg}$ \\
\hline Presión Parcial de O2 & $75-100 \mathrm{mmHg}$ & $49 \mathrm{mmHg}$ \\
\hline Presión Parcial de CO2 & $35-45 \mathrm{mmHg}$ & 7.40 \\
\hline $\mathrm{PH}$ & $7.35-7.45$ & $23 \mathrm{mEq} / \mathrm{L}$ \\
\hline $\mathrm{HCO} 3$ normal & $22-26 \mathrm{mEq} / \mathrm{L}$ & $45 \%$ \\
\hline FiO2 & - & 18 \\
\hline Coagulación (10/8/16) & $11.5-15.2$ & 1.27 \\
\hline Tiempo de trombina & & 45.8 \\
\hline INR & $20.0-40.0$ & \\
\hline T. parcial de tromboplastina & & \\
\hline
\end{tabular}

Fuente: Expediente clínico 


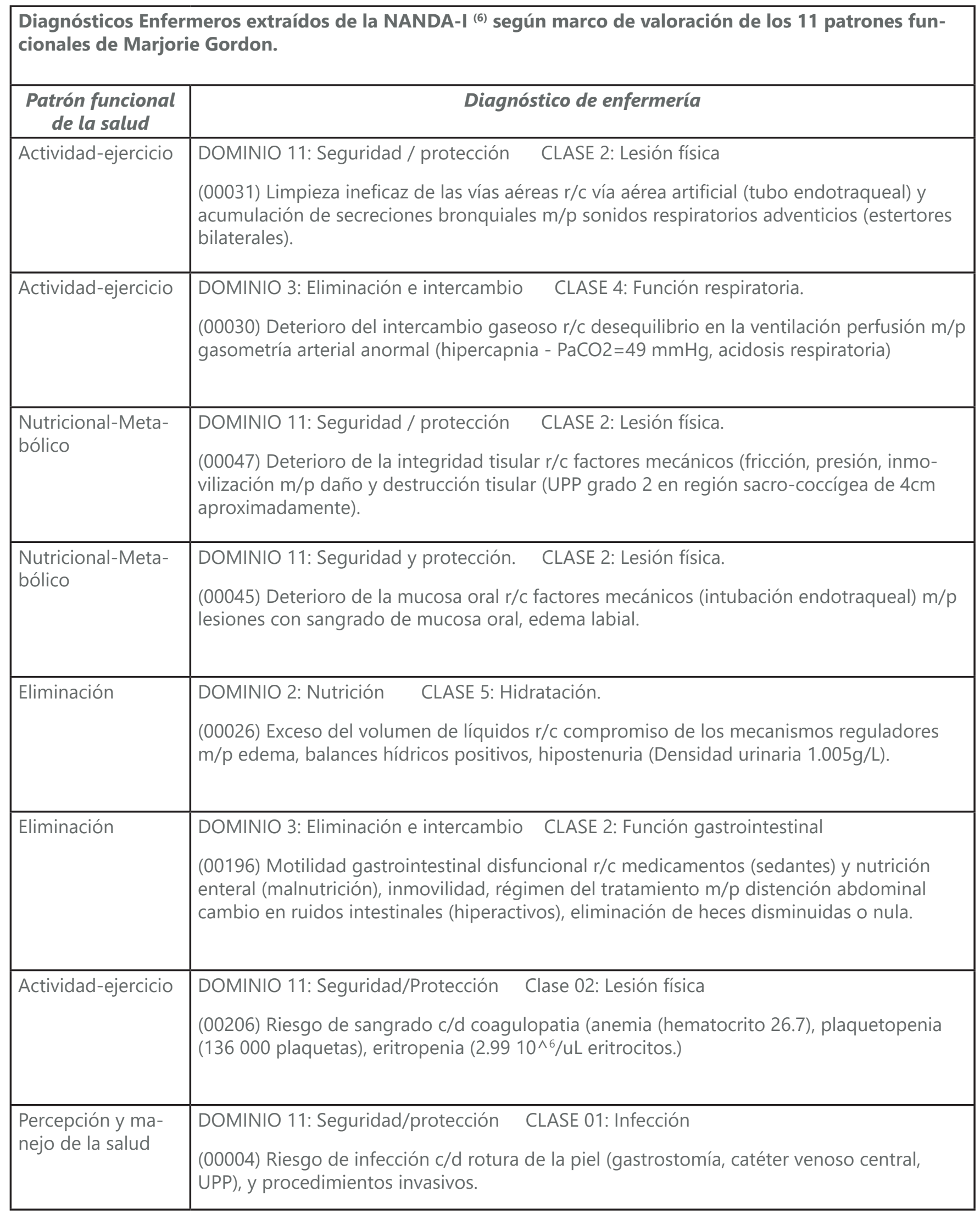



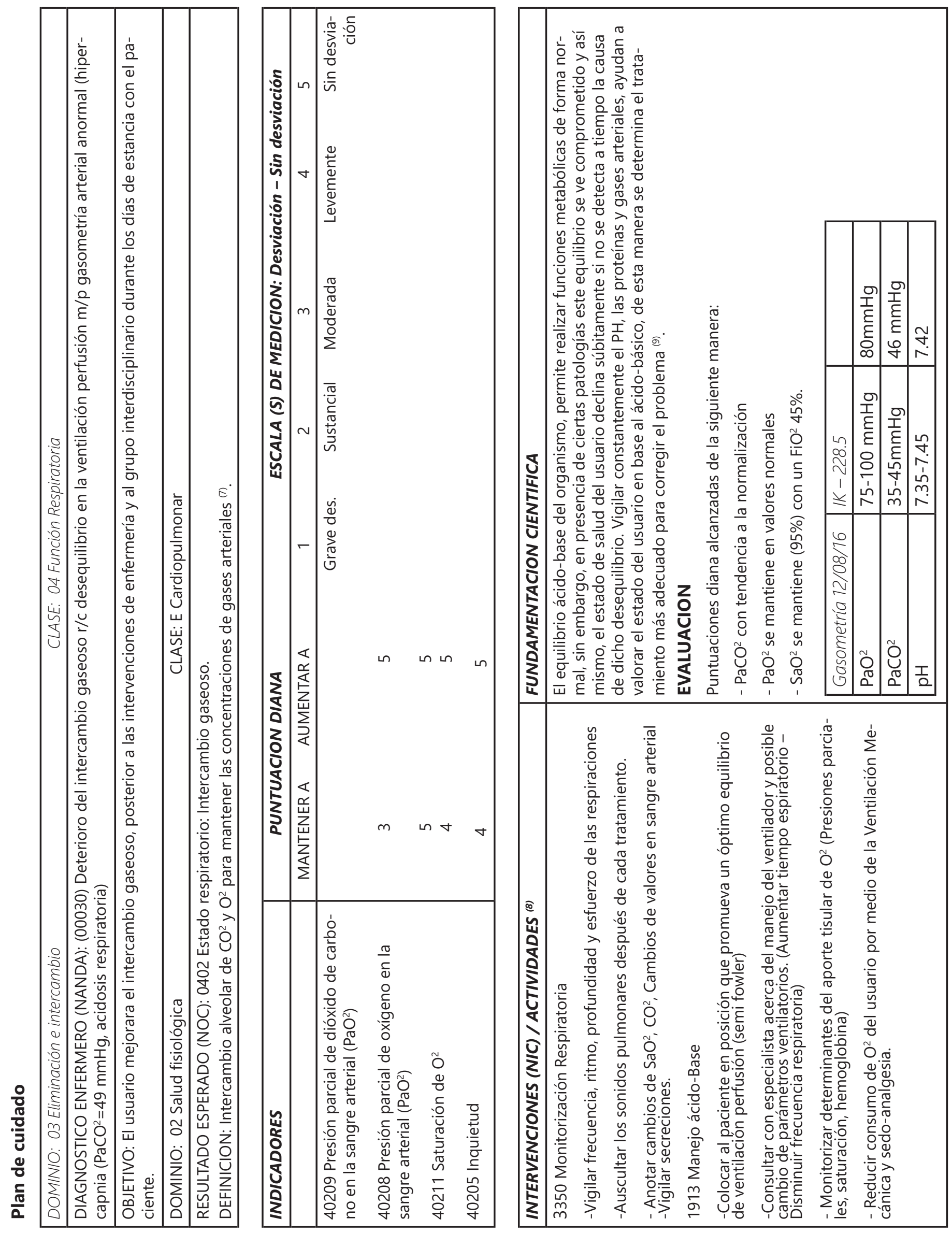

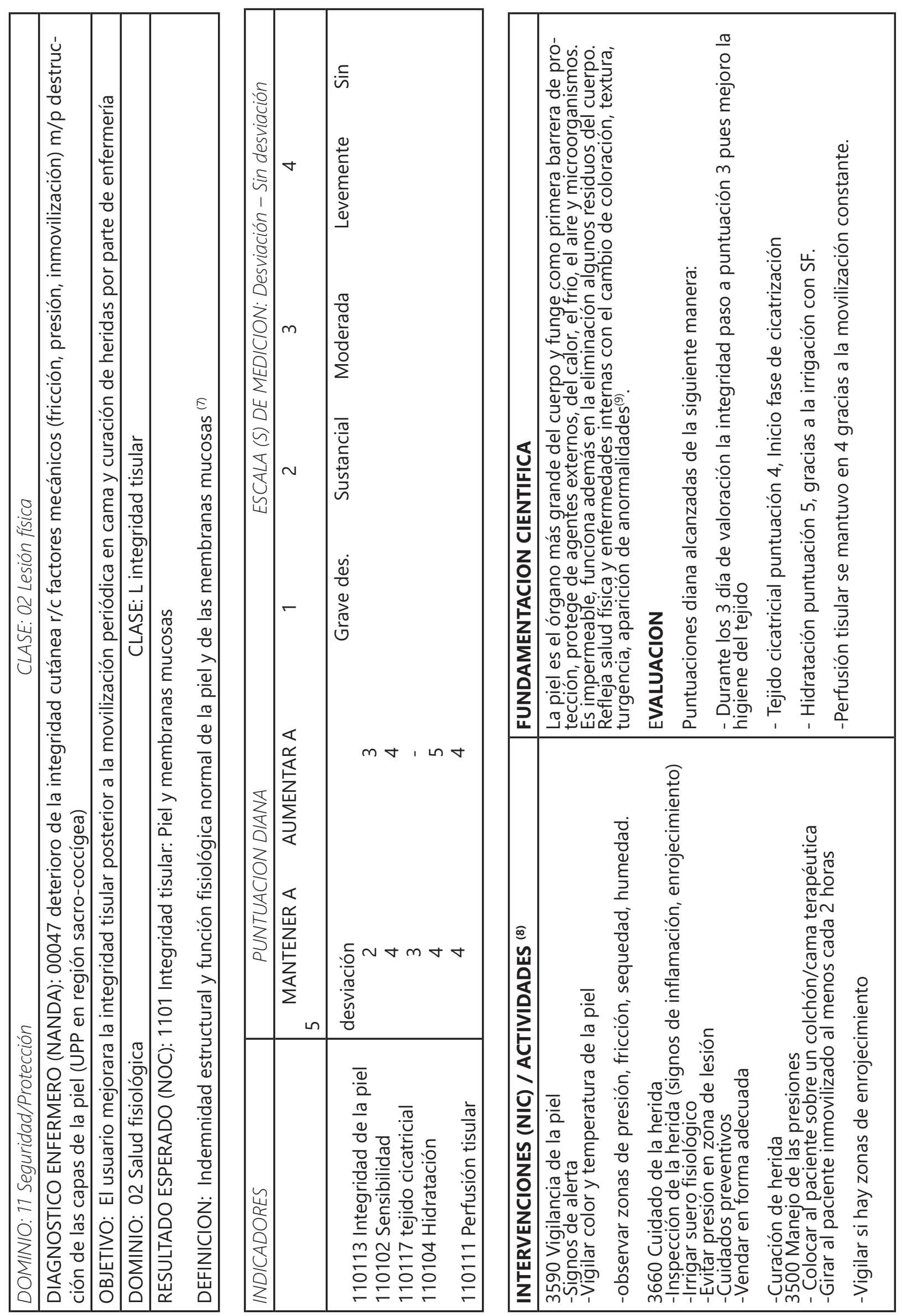


\section{CONCLUSIONES}

El estudio de casos es un método útil para analizar diferentes situaciones clínicas, identificar puntos fuertes y débiles en relación al cuidado establecido por enfermería y de esta forma plantear soluciones y estrategias para mejorarlo. La diferencia entre el estudio de caso y el caso clínico convencional, es la mirada fenomenológica donde se expone la relevancia para comprender la complejidad del cuidado en la práctica profesional, lo cual debe ser acotado por los límites de nuestro objetivo de estudio, y a su vez expandido según el contexto. De esta forma favorece la actitud reflexiva sobre situaciones de interés en la práctica, impactando positivamente en la conciencia de la complejidad y singularidad del cuidado, y al mismo tiempo de las consecuencias que podemos generar en quien lo recibe ${ }^{(3)}$.

Por otra parte, la valoración focalizada de enfermería permite identificar las necesidades de los usuarios y así mismo contribuir con el cuidado de estas, para ello es responsabilidad tanto ética como legal que el profesional de enfermería posea tanto conocimiento teórico como destrezas en las técnicas para brindar un cuidado humano y completo a los usuarios según la patología presente.

En el manejo del paciente critico influyen muchos factores que enfermería debe de considerar, ya que la identificación tardía de complicaciones en el estado de salud se correlaciona negativamente en el proceso saludenfermedad, lo cual genera una elevación en los costos de atención, tiempos de hospitalización prolongados, secuelas permanentes, e inclusive la muerte.

\section{REFERENCIAS BIBLIOGRAFICAS}

1. Reyes-Gómez E. Fundamentos de enfermería: Ciencia, metodología y tecnología. 2da ed. México: Editorial El Manual Moderno; 2015. 495 p.

2. Kozier B, Berman A, Snyder S, Erb G. Fundamentos de enfermería: conceptos, proceso y práctica. 8a ed. España: PEARSON EDUCACIÓN; 2008. 1620 p.

3.Orkaizagirre-Gómara A, Amezcua $M$, HuércanosEsparza I, Arroyo-Rodríguez A. El Estudio de casos, un instrumento de aprendizaje en la Relación de Cuidado. Index de Enf. 2014;23(4):244-9.

4. Rosa-Salazar V, Guirado-Torrecillas L, HernándezContreras M. Síndrome de vena cava superior como manifestación de carcinoma tímico. Arch. Bronconeumo. 2012: 48(10): 382-87.

5. Ibarra-Pérez C, Kelly-García J, Fernández-Corzo MA Guía diagnóstico-terapéutica: Tumores y masas del mediastino. Rev. Inst Nal Enf Resp Mex. 2001;14(3): 172-7.
6. Herdman TH. NANDA Internacional. Diagnósticos Enfermeros: definición y clasificación. 2015-2017. Barcelona: Elsevier; 2014. 512 p.

7. Moorhead S, Johnson M, Maas M, Swanson E. Clasificación de resultados de enfermería (NOC). 5a ed. España: Elsevier; 2014. 776 p.

8. Bulechek G, Bulecher H, Dochterman J, Wagner C. Clasificación de intervenciones de enfermería (NIC). 6a ed. España: Elsevier; 2013. 664 p.

9. Smeltzer S, Bare B, et al. Enfermería medico quirúrgica de Brunner y Suddarth. $12^{\mathrm{a}}$ ed. España: Editorial Lippincott Williams \& Wilkins; 2013. 1250 p.

10. Marriner A, Raile M. Modelos y teorías de enfermería. $6^{a}$ edición. España: Elsevier; 2008. 850 p. 